ICACCG2020 30-31 July, 2020, Ansal University, Gurgaon, India

International Journal of Technical Research \& Science (Special Issue)

\title{
OUTLINE STUDY AND DEVELOPMENT OF WASTE BIN AND WASTAGE RECYCLING SYSTEM IN INDIA
}

\author{
Abhishek Dimri, Apoorva Nautiyal, Dr. Arti Vaish \\ E-Mail Id: artivaish@ansaluniversity.edu.in \\ School of Engineering \& Technology, Ansal University, Gurgaon, India
}

\begin{abstract}
You can use tech for almost everything today-including removing the trash. With intelligent waste management technology, companies can track their trash more closely than ever before. Smart waste collection systems not only can help cut costs, but they can also help to reduce the environmental impact of your company. Garbage disposal has never been so high-tech, from garbage sensors to self-sorting garbage canisters. The Ebin waste management system is a smart waste container that uses a camera, sensors and artificial intelligence to automatically identify, sort and compress waste. When the tool determines the content, form and color of the waste, it is dispersed inside the container-plastics, paper or glass-into the appropriate bin. The waste is then compressed, so that the overall amount will be up to five times smaller. A self-sorting waste bin automatically notifies the waste disposal service once it has been filled in. It allows for optimization of collection routes to increase fuel efficiency. A waste management system not only addresses the issue of unsuitable waste sorting but it also monitors and sends data about the collected waste to an automated cloud. Your company will then use this knowledge to identify trends of customer use and decide more environmentally friendly alternatives for the product.
\end{abstract}

Keywords: Ebin waste management system, ebin, user-friendly technology.

\section{INTRODUCTION}

Waste management is just about the one thing every city government does for its citizens. Although the rates of operation, environmental effects and costs differ considerably, solid waste management is undoubtedly the most critical municipal operation and is a prerequisite for other municipal behavior. The amount of municipal solid waste (MSW), one of the most important by-products of an urban lifestyle, is growing even faster than the rate of urbanization as the world hurts to its urban future. Ten years ago, 2.9 billion urban residents generated approximately $0.64 \mathrm{~kg}$ of MSW per person per day ( 0.68 billion tons per year). The study estimates that these amounts are now supplying $1.2 \mathrm{~kg}$ per person per day (1.3 billion tons per year) to approximately 3 billion people. This is projected to grow to 4.3 billion urban residents by 2025 , producing around $1.42 \mathrm{~kg} /$ capita / day solid industrial waste ( 2.2 billion tons / year).

As the world begins to re-open it introduces a new way of life. Everyday activities and objects are reconfigured to adhere to protocols of social distance and provide a secure way to return to "business as normal" As a result, demand for products is that which improve safety in public places.

Bin-e identifies the form of waste automatically, too. It uses a proprietary method to classify the object, using artificial intelligence algorithms. It then segregates the waste into the appropriate fraction, compresses it and interacts with the consumer through an integrated screen. It also has the ability to monitor one or more devices via mobile app.

Contactless Bin-e is a new version of Bin-e, (a) device that will automatically sort and compress the recyclables, "To make waste management simple and effective it incorporates innovative AI-based identification, fill level control and data processing.

The Bin-e automatically detects segregates and compresses waste. This is based on a method of object recognition, which uses algorithms for machine learning and artificial intelligence. In addition, Bin-e has a fill control sensor and automatically notifies the waste disposal company when one of the bins inside is loaded. It has an Internet of Things connection.

Bin-e increases the amount of energy produced and reduces the amount of waste that goes into landfills. It turns waste management into an integrated framework to make the road to a circular, sustainable economy easier.

\section{INDIA'S CHALLENGES IN WASTE MANAGEMENT}

The laws regulating waste management in India are based on the values of "sustainable growth," "caution" and "polluter pays" Such standards enable municipalities and commercial enterprises to behave in an environmentally friendly and ethical manner.

The increase in waste generation as a by-product of economic development has led to various subordinate laws regulating the way waste is disposed of and dealing with waste generated under the Environment Protection Act, 1986 (EPA) umbrella legislation.

The country faces major waste management problem with rapid urbanization. More than 377 million urban residents live in 7,935 cities and towns, producing 62 million tons of solid municipal waste per year. The waste is collected just 43 million tons (MT), 11.9 MT is processed and $31 \mathrm{MT}$ is deposited in landfill sites. 
ICACCG2020 30-31 July, 2020, Ansal University, Gurgaon, India

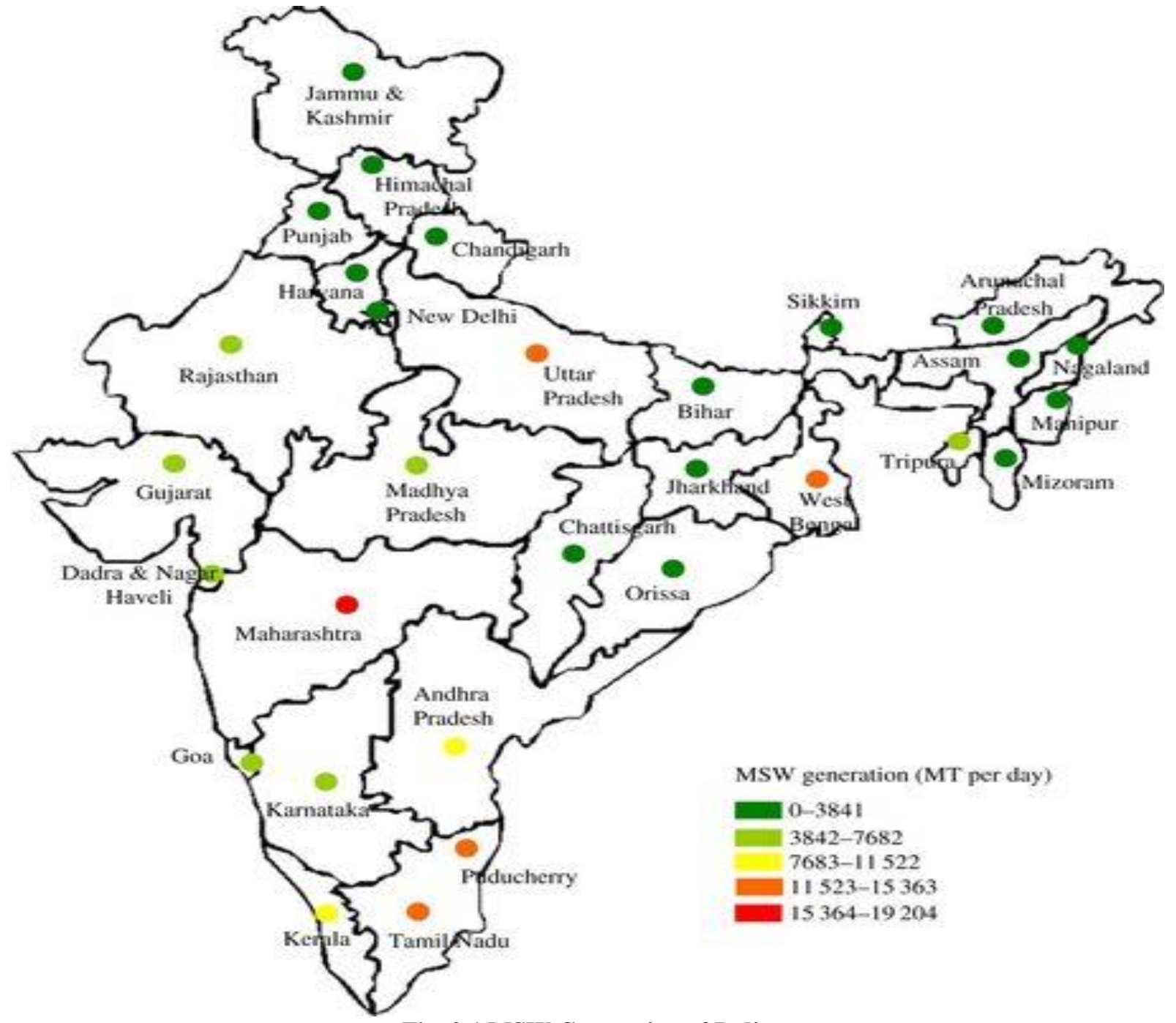

Fig. 2.1 MSW Generation of India

\subsection{Municipal Solid Waste}

$>$ MSW produced in India for the period 2001-2041

$>$ Urban waste power renewable energy in India 2009-2019

$>$ Residue recycling potential of leading states India 2016

$>$ Per State leadership in solid waste generation in India 2017

$>$ Indian solid waste treatment plants, by form 2019

\subsection{Plastic and Electronic Waste}

$>$ Plastic waste produced in India between 2001-2041

$>$ Plastic packaging industry market Size India FY 2010-2020

$>$ Share of plastics consumption India by sector in 2016

$>$ Sharing by nation of leading e-waste generators India 2016

\subsection{Biomedical Waste}

$>$ The volume of biomedical waste produced in India from 2007-2018

$>$ Common treatment facilities for biomedical waste, India 2008-2017

$>$ Biomedical waste treatment and disposal, India 2007-2018

$>$ Biomedical waste recycling market value in India 2010-2018

$>$ Biomedical waste recycling market volumes in India for 2010-2018

$>$ Biomedical waste recycling market share in India 2018 by industry

\subsection{Public Awareness}

$>$ Much of India's environmental problems for 2019

$>$ Policy suggestions for plastics reductions India 2019

$>$ Individual plastics reduction measures India 2019

$>$ Opinion on cleanliness complaint responsiveness by municipalities in India 2015-2019

$>$ Agreement on improper disposal of garbage since the 2019 Swachh Bharat Mission to India

DOI Number: https://doi.org/10.30780/specialissue-ICACCG2020/038 


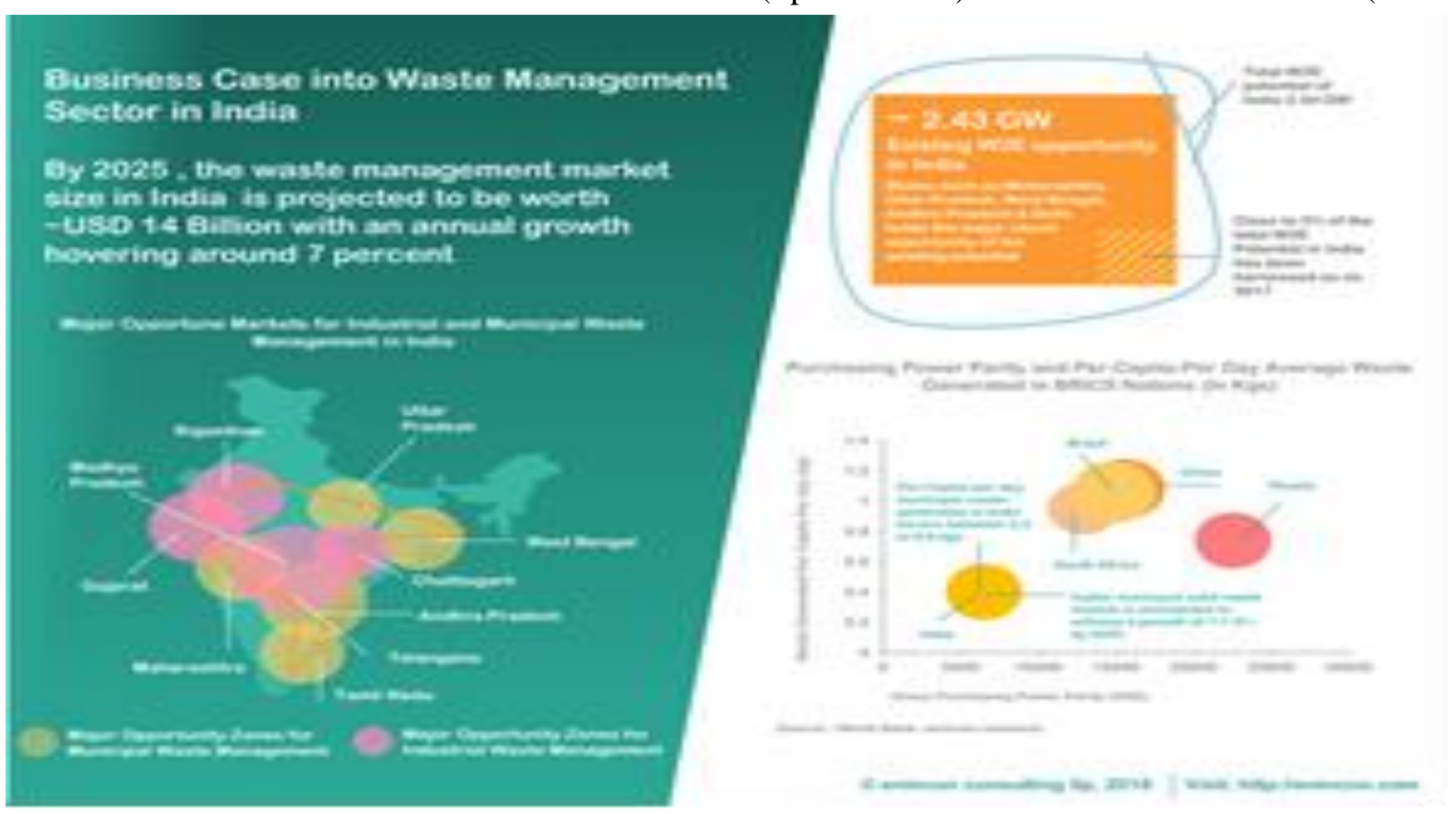

Fig. 2.2 Business Case into Waste Management in India

\section{METHODOLOGY}

We tried to provide the people with a complete solution, where smart waste management has to be smart on many levels. "To this end, we need to combine automated sorting, compression, fill level control and data processing into a single system, all of which enable A to transform waste management into an integrated acceleration of sustainability and the global transition to clean energy.

$>$ Throwing

$>$ Ai-Based Waste Recognition

$>$ Automatic Sorting

$>$ Plastic and Paper Compression

$>$ Fill Level Control

$>$ Data Processing in The Cloud

\section{RELATED WORK}

There are smart bins worldwide which provide an intelligent way to communicate with their users. One clear example of such a device is the renewed pods. Renew pods offer news, information, and recycling bins for the Commuters in London. Renewed connected pods launched at 25 locations in the city of London. Renew has recently deployed 25 of its futuristic to street pods across the region. For more than six years the Renew model has a dual feature in service apart from acting as a recycling depository; the pods are also a mobile hub with LCDs on both front and back, providing passengers with the latest financial updates and world news.

\section{PROPOSED DESIGN OF THE SYSTEM}

System users are very vividly, depositors and collectors are the two main users of the system. The smart bin has four important components, including the arduino board, the servo motor, the ultrasonic sensor and the android device. The Firebase cloud services are used to authenticate device users. The ultrasonic sensor measures the bin's fullness, while the servo motor secures the bin by locking it from the inside and unlocking the bin with the smart bin's system architecture and its various components for authorized users. For this system there are three basic component categories, which include IoT, Frontend and Backend applications IoT refers to the smart bin itself, with the sensors and IoT modules required. The front end refers to the Android applications that are mounted on the smartphone of users. These include application by the depositor and the collector. The backend refers to the PHP files that are stored on the server with the ability to fire certain SQL queries required for transactions on the depositor and collector tables of the database.

\subsection{Frontend - Android application}

Android Apps This device has three types of android devices. The depositor app, collector app, and the smart bin app that needs to be installed inside the smart bin's android device. First, the depositor application is developed with a brilliant user interface that makes several options available to users in a very presentable way. Some of these options include displaying the location of the bins across the area, displaying information about the user profile, displaying the status of the items he / she deposited. Secondly, the collector application is developed with options suitable for the collectors of e-waste such as participating in active auctions, Checks the auction result and also 
updates the weight of the items deposited by the depositors. Thirdly, the smart bin app that is part of the bin itself (installed in the bin's android device) provides users with a UI to log in to the bin. Apart from this, collecting data from the microcontroller (Arduino) and checking the bin status is also needed. If the bin is complete it sends the server an HTTP request to start an online bidding session.

\subsection{Server (Backend)}

The server is the data store house needed to make this system functional. It stores the databases and the necessary PHP files for accessing data from them. The front end makes http requests to the backend, and the server responds with the correct answer. Any of the tables contained in the database are consumer information tables, collector information, bin information table, bidding table. Because relational databases offer competitiveness control, online bidding sessions are fairly easy to perform even if multiple bidders place their bid at the same time. The server also has a Cron Job scheduler which will automatically generate http requests at defined intervals. It is especially useful when announcing bidding session results.

\section{OVERALL DESIGN}

The smart dustbin consists of different sections for all kinds of waste disposal which includes:

$\begin{array}{ll}> & \text { Plastic Waste } \\ > & \text { Metal Waste } \\ > & \text { Liquid Waste } \\ > & \text { Paper Waste } \\ > & \text { Organic Wastes }\end{array}$

\section{HARDWARE SPECIFICATIONS}

Sensor for garbage segregation Different types of sensors used for successful functioning of garbage segregation are reviewed in this chapter. Sensors are the basic components of the Automatic smart trash bin system in which the waste is detected, sensed, and separated. The rapid development in the same stream of integrated circuits and other developments has expanded the use of smart sensors to produce fast and reliable results with less energy preservation. The AK970 IR sensor is used for human sensing purposes. This sensor's working voltage varies between $1.71 \mathrm{~V}$ and $3.63 \mathrm{~V}$. These passive Infrared sensors can perform human detection within the range of 5-12 $\mathrm{m}$. The second main sensor used to detect waste in a running system is an ultrasonic sensor. By transmitting a sound pulse, this ultrasonic sensor tests the distance of the garbage and the time delay obtained back as an echo signal. Following the ultrasonic sensor, the conveyor belt moves the waste slowly across the metal and plastic sensors and starts the segregation process. Electromagnetic waves are produced in metal sensor with high frequency and high ringing stress. When a metal is felt, the coil is detuned; this in effect decreases the segregation. Plastic sensors are used to separate plastic from paper and vegetable peel according to the metallic sensor. A plastic sensor 's main working principle is obtained by varying the capacitive voltage between the pairs of plates by varying the dielectric mean. Thus plastic sensor completes biodegradable segregation function and no biodegradable waste and vegetable peeling. MyRIO is used as the platform to automatically code the sensors and motors.

\section{WORKING MECHANISM}

The garbage segregation process is implemented in three ways, the recycling process may be recommended for the effective sorting system based on the type and condition of the waste. This system relies on proximity sensors in the device and plastic material sensor for the object's presence. The plastic sensor determines plastic levels according to the resonant frequency of each plastic material. The molecules of the various plastic material are excited by the tuning of laser diodes for specific plastic resonant frequencies and are thus detected. The waste is isolated by the combination of proximity sensors and plastic sensors, based on its degradability. It offers us the option for plants to dispose of the non-degradable waste as recyclable and degradable as manure. That's also significantly affordable and stable, It also reduces the spread of germs through garbage, since the water is recycled regularly to plants and the setup is closed. Once any of the small bin is full, the larger bin will not open until the waste is cleaned, thereby ensuring no garbage overflow. The challenges faced is that the setup is larger than the usual trash bins, and all the sensors and motors connected to a single myRIO are implemented. Benefits Compression wastes can reduce emissions. The principal benefit of recycling is the turn of waste into gold. Compression reduces earth resource consumption. The setup of the automation circuit is simulated and the operation results are generated. Successful execution to implement the automated smart dustbins can avoid the accumulation of the garbage on the roadside and thereby curb the formation of microorganisms which in turn prevent the diseases. Pollution can be prevented and the street animals can be avoided for garbage consumption

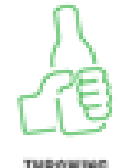

THล0อง

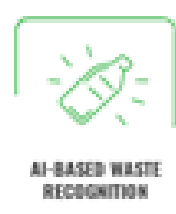

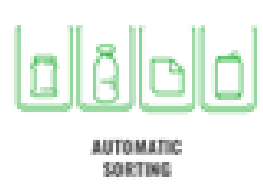

Fig. 8.1 Working Scheme

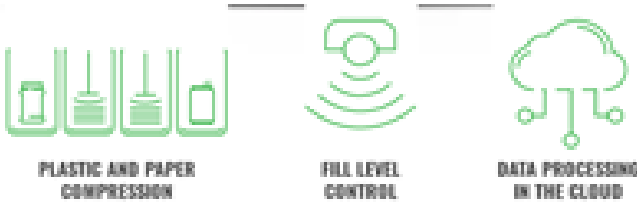


ICACCG2020 30-31 July, 2020, Ansal University, Gurgaon, India

International Journal of Technical Research \& Science (Special Issue)

8.1 Depositor Android Application

$>$ The application has the following options: to determine the location of bins in different locations, to determine their reward points, to determine the status of their deposited items, to scan the QR codes of the bags they deposit in.

\subsection{Smart-Bin}

> As mentioned above, several IoT components as well as a device that has an android application for depositors and collectors of authentication and also creates bidding sessions based on the bin status (full or empty).

\subsection{E-Waste Collector Android Application}

$>$ Collector can use his/her application for the following purposes: checking the availability of active auctions, checking the results of the concluded auctions and updating the weights of the depositors' items.

\subsection{Problem of ebin in India}

$>$ Smart bins which are previously installed by govt are expensive but they cannot rid the valley of roadside trash.

$>$ The store capacity of these bins are very less.

$>$ Majority of people are unaware of the rectangular box on the streets was really a dustbin.

\subsection{How e-bin Helps to Solve these Problems}

$>$ E-Bins are cheaper and more effective than smart dustbin installed by gov.

$>$ Compression technology helps to store more amount of waste products.

$>$ Eco-friendly.

$>$ Saves time and human effort.

\section{SWOT ANALYSIS OF WASTE MANAGEMENT}

$>$ Highly skilled labor force by effective educational programs and learning. Waste Management invests huge resources in its employees' training and development resulting in a workforce that is not only highly skilled but also motivated to achieve more.

$>$ Highly successful at Go To Market strategies for its products.

$>$ Automation of activities has brought quality consistency to waste management products and allowed the business to scale up and scale down according to market demand conditions.

$>$ Superb Performance in New Markets - Waste management has developed resources to reach and make success in new markets. The expansion helped the company develop new revenue sources and diversify the impact of the economic cycle in the markets in which it operates.

$>$ Successful track record of developing new products - product innovation.

$>$ Good Brand Portfolio-Waste Management has invested in developing a large brand portfolio over the years. Waste Management's SWOT analysis only underlines the fact. A portfolio of products can be incredibly useful if the company wishes to move into new product categories.

$>$ Strong returns on capital expenditure - Waste management succeeds fairly in introducing new projects and produces reasonable returns on capital expenditure by creating new revenue streams.

$>$ Solid distribution network - Waste Management has developed a robust distribution network over the years that can meet much of its business potential.

\section{WEAKNESS OF WASTE MANAGEMENT - INTERNAL STRATEGIC FACTORS}

Weakness is where Waste Management can be improved. Strategy is about making choices and weaknesses are areas where a company can improve its competitive advantage and strategic positioning using SWOT analysis.

The company was unable to address the new entrants' challenges in the segment and lost small market share in the categories of niches. Waste management must develop internal input systems directly from on-the-ground sales team to overcome these challenges.

Research and Development investment is below the sector's fastest growing players. While waste management spends more than the industry average on research and development, it has failed to compete with the industry's leading players in terms of innovation. It has come across as a mature firm looking forward to bring out products based on tested features in the market. Not very good in merging businesses with diverse work cultures. As mentioned earlier, although Waste Management succeeds in integrating small businesses, it has its share of failure to merge companies with different working culture. The productivity ratio and Waste Management's Net Contribution rate are below industry average.

The advertising promotion has left much to be desired. Even though the product is a sales success, its positioning and unique selling proposition is not clearly defined which can lead to competitors attacks in this segment. There are holes in the company's selection of goods offered. That lack of choice can give the market a foothold to a new competitor. 
ICACCG2020 30-31 July, 2020, Ansal University, Gurgaon, India

International Journal of Technical Research \& Science (Special Issue)

ISSN No.:2454-2024 (online)

Not very good forecasting of product demand leading to higher rate of missed opportunities compared with its competitors. One of the reasons why the inventory of days is high relative to its rivals is that waste management is not very successful when it comes to demand forecasts. keeping higher inventory both in-house and in channel

\section{OPPORTUNITIES FOR WASTE MANAGEMENT}

\subsection{External Strategic Factors}

The core competencies of an organization can be a success in similar field of other products. Could be a comparative example-GE healthcare research helped it develop better oil drilling machines.

Stable free cash flow offers incentives for investment in neighboring segments of goods. With more cash in the accounts, the company will invest in new technology and in new segments of goods. This will give Waste Management a window of opportunity in other product categories.

New policies on the environment - The new opportunities will create a level playing field for all industry players. It represents a great opportunity for waste management to drive home its new technology advantage and gain market share in the new category of products.

New Online Channel customers - The company has invested huge sums of money in the online platform over the last few years. This investment opened new Waste Management sales channel. In the next few years the company can take advantage of this opportunity by better knowing its customers and by using big data analytics to serve their needs.

The new tax reform will have a huge effect on the way business is conducted, which will open up new opportunities for existing companies like Waste Management to improve their productivity.

Business growth would dilute the benefit of the company and allow Waste Management to improve its profitability as compared to other competitors. The new technology gives Waste Management an opportunity to pursue competitive pricing strategies in the modern market. It will allow the company to maintain its loyal customers with great service and will attract new customers through other value-oriented proposals.

Opening up new markets by government agreement - adopting new technology standard and government free trade agreement has given Waste Management an opportunity to enter a new emerging market.

\subsection{Threats Waste Management Facing}

\subsubsection{External Strategic Factors}

As the company operates in numerous countries, it is exposed to currency fluctuations, particularly in view of the volatile political climate in numerous worldwide markets. Changing consumer buying behavior from the online channel could represent a threat to the existing supply chain model driven by physical infrastructure.

Growing strengths of local distributors also pose a threat in some markets, as competition is paying the local distributors higher margins. In the medium to long term, new technologies developed by the competitor or market disruptor could present a serious threat to the industry.

Intense competition - In the last two years, stable profitability has increased the number of players in the industry which has put downward pressure not only on profitability but also on overall sales. Increasing pay levels, particularly movements like $\$ 15$ an hour and rising prices in China, can lead to severe pressure on waste management 's profitability

New environmental regulations could pose a threat to certain existing product categories under the Paris Agreement (2016). That movements in the American economy towards isolation will lead to similar reactions from other governments that have a negative effect on foreign sales..

\subsection{Further Development of this Project}

$>$ Fixing mechanism

$>$ The collector bins can be separately underground for efficient disposal.

$>$ Use of a hydraulic system to fill garbage truck waste materials.

$>$ The degradable waste collected can then be converted into compost manure.

$>$ We can use a smart card that includes ID no for the billing system and people can use the card with the bin by scanning it.

\section{CHALLENGES AND OPPORTUNITIES ASSOCIATED WITH WASTE MANAGEMENT IN INDIA}

Solid waste management (SWM) is a major problem for many urban local bodies (ULBs) in India, where urbanization, industrialization and economic growth have led to increase per capita generation of municipal solid waste (MSW) [1]. Efficient SWM is a big challenge in densely populated cities. Sustainable development in a country with rapid population growth and improved living standards in India is made more difficult because it is a diverse country with many different religious groups, cultures and traditions.

SWM systems in India have remained relatively unchanged despite significant development in the social, economic, and environmental areas. The informal sector plays a key role in the extraction of waste value, with around $90 \%$ of the residual waste currently being dumped instead of properly landfilled [2]. There is an urgent need to move towards more sustainable SWM, and new management systems and waste management facilities are needed here. 
Current SWM systems are inefficient, with the effect of waste on public health, the environment and the economy [3]. The Indian waste management and handling rules were adopted by the Ministry of Environment and Forests[4], while enforcement is variable and restricted.

\section{REFERENCES}

[1] http://fernfortuniversity.com/term-papers/swot/1433/221-wastemanagement.php\#: :text=SWOT\%20analysis\%20is\%20a\%20strategic,in\%20its\%20current\%20business \%20e nvironment.

[2] https://royalsocietypublishing.org/doi/10.1098/rsos.160764" The front end makes http requests to the backend, and the server responds with the correct answer. Any of the tables contained in the database are consumer information tables, collector information, bin information table, bidding table. Because relational databases offer competitiveness control, online bidding sessions are fairly easy to perform even if multiple bidders place their bid at the same time".

[3] https://streetfurniture.com/product/escola-bin-enclosure.

[4] https://en.wikipedia.org/wiki/Waste_management_in_India" SWM systems in India have remained relatively unchanged despite significant development in the social, economic, and environmental areas. The informal sector plays a key role in the extraction of waste value, with around $90 \%$ of the residual waste currently being dumped instead of properly landfilled"

[5] Saurabh Dugdhe, Pooja Shelar, Sajuli Jire and Anuja Apte" Efficient Waste Collection System 2016 International Conference on Internet of Things and Applications (IOTA) Maharashtra Institute of Technology, Pune, India 22 Jan -24 Jan,2016.

[6] Dr. N. Sathish Kumar\#1, B.Vijayalakshmi\#2, R. Jenifer Prarthana\#3, A. Shankar\#4 "IOT Based Smart Garbage alert system using Arduino UNO”, 2016 International conference on recent advances in Internet of Things,8-9 June 2016.

[7] Md. Abdulla Al Mamun, M. A. Hannan, Aini Hussain "Real Time Solid Waste Bin Monitoring System Framework Using Wireless SensorNetwork" International conference 37- 40, 2009.

[8] Mohd Helmy Abd Wahab and Aeslina Abdul Kadir "Smart Recycle Bin A Conceptual Approach of Smart Waste Management with Integrated Web based System”. IEEE 2014. 